\title{
Does the use of open, non-anonymous peer review in scholarly publishing introduce bias? Evidence from the F1000Research post- publication open peer review publishing mode ${ }^{1}$
}

As part of moves towards open knowledge practices, making peer review open is cited as a way to enable fuller scrutiny and transparency of assessments around research. There are now many flavours of open peer review in use across scholarly publishing, including where reviews are fully attributable and the reviewer is named. This study examines whether there is any evidence of bias in two areas of common critique of open, non-anonymous (named) peer review - and used in the post-publication, peer review system operated by the openaccess scholarly publishing platform F1000Research. First, is there evidence of potential bias where a reviewer based in a specific country assesses the work of an author also based in the same country? Second, are reviewers influenced by being able to see the comments and know the origins of a previous reviewer? Based on over 4 years' of open peer review data, we found some evidence, albeit weak, that being based in the same country as an author may influence a reviewer's decision, while there was insufficient evidence to conclude that being able to read an existing published review prior to submitting their review encourages conformity. Thus, whilst immediate publishing of peer review reports appears to be unproblematic, caution may be needed when selecting same-country reviewers in open systems if other studies confirm these results.

\section{Introduction}

The trend towards more open and collaborative knowledge practices ('open science') has led to an increasing number of journals and publishing outlets offering 'open peer review' to various degrees [1-3].

F1000Research today perhaps offers one of the most comprehensive versions of 'open peer review'; across all its publishing platforms, a post-publication peer review model is provided alongside invited, open peer review. Each article, after initial editorial and objective checks (e.g. for ethical approval; plagiarism; and inclusion of underlying data) is published before peer review. The subsequent peer review process, notable in the debate about the value of open peer review, publishes the identities and affiliations of all peer reviewers alongside their narrative report. The publication process is iterative ('continuous publishing'), with authors able to respond directly to their reviewers' comments by providing a revised article version, linked to the previous version to preserve the revision history.

To keep the process rapid and iterative, peer reviewer reports are published (following an initial check) as soon as they are submitted thus allowing the possibility for subsequent reviewers to read already published reports before submitting their own review. Additionally, the post-publication peer review model is intended to shift the role of peer review in scholarly publishing from its traditional focus on selecting content for publication, to one that helps to shape the research to be the best it can be - and providing this openly, allows readers to reflect on the development of a piece and consider any reviewer critique. Nevertheless, open peer review introduces the potential for specific types of reviewer biases and since peer review of scholarly output continues to provide an important quality control function [4], it is

\footnotetext{
${ }^{1}$ Thelwall, M., Allen, L. Papas, E., Nyakoojo, Z., \& Weigert, V. (in press). Does the use of open, non-anonymous peer review in scholarly publishing introduce bias? Evidence from the F100OResearch post-publication open peer review publishing model. Journal of Information Science
} 
important to examine the benefits and potential biases that newer open systems of peer review may introduce.

There are many types of potential bias described within peer review processes and practices operated by grant funding agencies and in scholarly publishing, including according to: author/grant applicant demographic characteristics [5-8]; author-reviewer speciality differences (cognitive distance)[9]; country of affiliation/article country of origin[10], and perceptions of authors' institutional prestige[11]. Perhaps because such studies revealing potential biases have been typically tested on the single-blind model (with author/grant applicant identity known by reviewers), there remains a widespread belief that double-blind peer review introduces the 'least' bias and is superior to open peer review approaches[1214]. However, beliefs about the merits of open peer review are supported by limited empirical evidence and based upon survey and attitudinal research[15].

From studies providing empirical insights into open peer review, there is evidence that providing peer review transparency has a minimal effect on review quality. Two randomised control trials of The BMJ did not find quality differences between the reviews provided when reviewers knew that their identities would be revealed, compared to blinded review [16] or when reviewers were told their reviews may be published online[17]. A study of a psychology journal found that reviews tended to be more carefully written when they were intended to be openly available[18]. A systematic review of research into peer review for biomedical journals found nine studies into concealing author or reviewer identity, but no conclusion could be reached overall about how this influenced review quality[19]. However, a recent study of a large volume of articles submitted to Elsevier journals concluded that using an open peer review process does not compromise the quality and participation in the process, at least when reviewers are able to protect their anonymity[20], although The BMJ reviewers were less willing to review when their identities would be revealed[16]. While there is some evidence that open peer review might deter reviewer acceptance rates[15], there is limited empirical evidence about the effect that revealing reviewers' identities and affiliations and 'signed peer review' policies, has on peer review in practice.

As part of the efforts to provide a firmer evidence base to support decision-making around the use of open peer review, this article investigates a post-publication peer review process, accompanied by fully open and named peer review (used by F1000Research across all its publishing platforms) for evidence of potential biases in several dimensions. First, since reviewers and authors know each other's identity, is there any evidence that reviewers are more generous to authors who they know or are likely to meet or collaborate with at some point, for example if they are based in the same country? Reviewers are not allowed to be from the same institution as any of the authors. Social factors have been shown to influence peer review decisions in other contexts (regional bias:[21]), and so this is a plausible hypothesis, although there are other types of social bias are not tested for here (e.g., institutional prestige). Second, since reviewers can view and read other reviewer reports before submitting their own for the same article, they may be influenced by what they read (either consciously or subconsciously) and even use the existing report to reduce their own workload. This influence may be greater perhaps if the published reviewer is an established figure within a field. This study investigates evidence for these two types of bias: whether author and/or reviewer country affiliation influences reviewer comments and decisions; and whether the availability of previous reviewer reports influence the comments and decisions of subsequent reviewers. 
For academic peer review to be as effective and valuable to scientific discourse as possible, it is essential that potential biases are identified and, as far as possible, their effects mitigated, managed or eliminated. It is also important that scrutiny of newer models is matched with scrutiny of existing and established models.

\section{Methods}

\section{Data}

The data consists of the text and judgements of all reviews of version 1 outputs (i.e., before any revisions in response to reviewers) from F1000Research [22] published on the F1000Research platform between July 13, 2012, and July 8, 2019. Reviews were included for all types of articles submitted for peer review. Since the focus is on reviewer bias, there does not seem to be an obvious reason why this should differ between article types. Only reviews posted to first versions of articles were included. This is because reviews after the first version are more likely to give a positive verdict as the authors have revised their article in response to the reviewers' suggestions. Each reviewer report contains mandatory text, and a judgement of "Approved" (no changes or a few small or cosmetic changes), "Approved with reservations" (not fully sound in the current version), or "Not approved" (fundamental flaws and the work overall is poor quality; the authors are still encouraged to revise their article to respond to the concerns raised). Prior to mid-2013, it was possible for a reviewer to submit an overall assessment without any accompanying narrative, however this policy has been revised and now all peer review reports on version 1 must include both a narrative and assessment. All peer review reports and assessments are made public alongside the published article, regardless of whether it is 'approved' or not - the approval status does not affect the decision to publish, but determines when an article is considered to have 'passed peer review' and is therefore sent to indexers such as PubMed and Scopus.

For the 30 countries with at least 20 articles there were 2030 articles altogether, with 2553 articles from all 79 countries combined. A breakdown by national affiliation is in the first table. The reviewers were from the same 79 countries (presumably authors are asked to review other contributions), but with US researchers slightly over-represented amongst reviewers (29\% of articles but $34 \%$ of reviews). The articles are given ad-hoc author keywords (e.g., "moral growth mindset, growth mindset, reliability, validity, moral development") but not subject classifications and so it is not possible to report which subjects dominate F1000Research.

\section{Author and reviewer affiliation bias tests}

For the author/reviewer affiliation tests, only the country of the first author's affiliation was considered for multi-author papers, since the first author has typically provided the largest range of contributions to the published work. Although authorship can sometimes be misleading [23], empirical evidence suggests that the first author tends to be the most substantial contributor in all broad academic fields, even those with senior last author or alphabetical norms[24]. Thus, for the purposes of this analysis, the first author location was used as a proxy for the location of the whole authorship team. The first affiliation of the first author was used to identify the country. When authors have more than one affiliation, it seems likely that their main affiliation would be listed first.

In terms of potential reviewer-author biases, these could occur due to authors other than the main or first author and for affiliations of the authors other than the first author's 
main affiliation. These potentials were ignored for the test on the basis that they seem likely to be weaker biases, if they exist. If either of these biases exist, then they would reduce the statistical power of the test.

Only the first peer review report was assessed (for the affiliation tests; all were used for the previous review bias tests) to avoid non-independence for statistical tests due to multiple reports (by different reviewers) on the same article. Multiple reports by different reviewers on the same article are not statistically independent because different judgements of the same article are likely to agree more than different judgements on different articles. Author and reviewer national affiliations were judged from the country of their affiliation, or first affiliation if multiple affiliations were declared. Decisions were coded on the following simple numerical scale to allow averaging.

- 1: Not approved

- 2: Approved-with-reservations

- 3: Approved

Non-parametric Mann-Whitney $U$ tests were used to analyse author/reviewer affiliation bias since the data does not follow the normal distribution (from a Q-Q plot, although its skewness -0.66 and excess kurtosis -1.42 are reasonable). A hypothesis testing approach was used because the no-bias null hypothesis is reasonable and the purpose of the paper is to detect deviations from this.

The three-point scale is not well suited to the purpose of this paper because it is coarse-grained and few articles are given a status of 1 , so in practice it is almost binary. The scale was used in the absence of an alternative and in recognition of the need to test for bias in any way possible.

The bias test assumes that if reviewers give higher grades to authors from the same country than do international reviewers of the same articles, then there is reason to suspect national biases. There are other possible explanations of higher grades from same country reviewers, however. For example, reviewers from the same country might have more expertise in the subject area of the article (because there are national variations in research specialisms) and give better judgements that are, for some reason, more positive. This seems less likely than same country bias, however.

\section{Previous review bias tests}

Some reviewer reports mentioned previous reviewer reports, for example to say that they would not repeat points made in them. Such reports were identified by text searches for the strings, "Referee", "referee", Reviewer", and "reviewer", followed by a manual check of whether the term was used to refer to comments posted by a previous reviewer about the same article. This produced 143 examples of reviewers explicitly mentioning a previous reviewer report.

Reviewer reports on a single article were ordered using their identifiers, which are allocated consecutively. The published time of each report was then used to calculate the number of days between reports. A reviewer report is typically published rapidly, often on the same day that it is submitted, although occasionally the editorial team may need clarifications first. If two reports are posted on the same day then it is unlikely but not impossible for the reviewer from the second report to have seen the first report before writing and submitting their own. Nevertheless, the longer the time period between the first report appearing and the second report being published, the more likely it is that a subsequent reviewer had seen a prior report[25]. This is because older reports would have 
been more likely to be available to view when the subsequent reviewer first accessed the article to read, print, or download.

Agreement rates were calculated for each report (after the first). For this, the fraction of all previous reports that they agreed with was calculated. For example, if the first and third reviewer assigned an 'Approved' status, and the second reviewer assigned 'Not approved', then the second reviewer would have an agreement rate of $0 / 1=0$ (disagrees with the first reviewer) and the third reviewer would have an agreement rate of $1 / 2=0.5$ (agrees with one of the two prior reviewers).

Statistical tests, as described below, were used to assess the likelihood of bias due to reviewers being influenced by reading prior reviews. Non-parametric tests were used because agreement rates were not normally distributed.

\section{Results and discussion}

While overall only $5 \%$ of articles were assigned a 'Not approved' status during the peer review process, around a third of articles submitted to F1000Research (36\% in Jan-Oct 2019 from the authors' knowledge) do not adhere to policies and therefore do not proceed past the initial editor checks and are therefore not sent for peer review; this is similar to estimates of 35\%$40 \%$ overall reject decisions for academic journals, although $10 \%-15 \%$ lower for open access and health/biomedical journals[26.27]. Nevertheless, since $95 \%$ of the decisions analysed here are either 'approved' or 'approved with reservations', the analysis below primarily concerns whether the reviewer had reservations about a document that they otherwise broadly approved. This is an important distinction in terms of informing both authors and readers (until a new version is posted) about whether a paper is sound and therefore any biases in this stage are potentially important.

\section{Do author and reviewer national affiliations influence peer review judgements?}

The first area of potential bias is whether peer reviewers might be inclined to provide a favourable review to authors based in the same country, especially when their identity will be revealed. As background for this, the average judgements were analysed based on the national affiliations of authors and reviewers separately. This gives context about the average perceived quality of submissions and the average strictness of reviewers, by country, as well as the country-level relationship between the two. Reviewer same-country bias, the final test, would manifest in deviations from the average judgement of other reviewers for papers from a country, but could be caused by reviewers from that country always being more generous, than other reviewers, irrespective of the origins of the articles reviewed. Hence the background comparisons are important.

First, do authors from different countries receive different average judgements? Average judgements for articles varied with first author affiliation country to some extent (Figure 1). For example, decisions are statistically significantly more positive for the UK and USA than for India. This aligns with previous research showing that there are international differences in peer review outcome for authors from different regions [21] and in the average citation impact of academic research[28], although it is influenced by collaborations with higher income and research-intensive countries[29]. The main reasons cited for this potential inequality in research intensity and citation impact between countries include differing levels of financial support for research infrastructure, limited availability of mentors and experienced researchers, and the migration of highly skilled researchers to better-resourced economies[30]. 


\section{Author country}

Malaysia

Denmark

Pakistan

Belgium

Bangladesh

Sudan

Nigeria

New Zealand

Russian Federation

Thailand

Colombia

Egypt

Japan

Brazil

Sweden

Nepal

China

Iran

Spain

The Netherlands

Italy

France

Switzerland

Indonesia

Germany

Canada

Australia

India

UK

USA

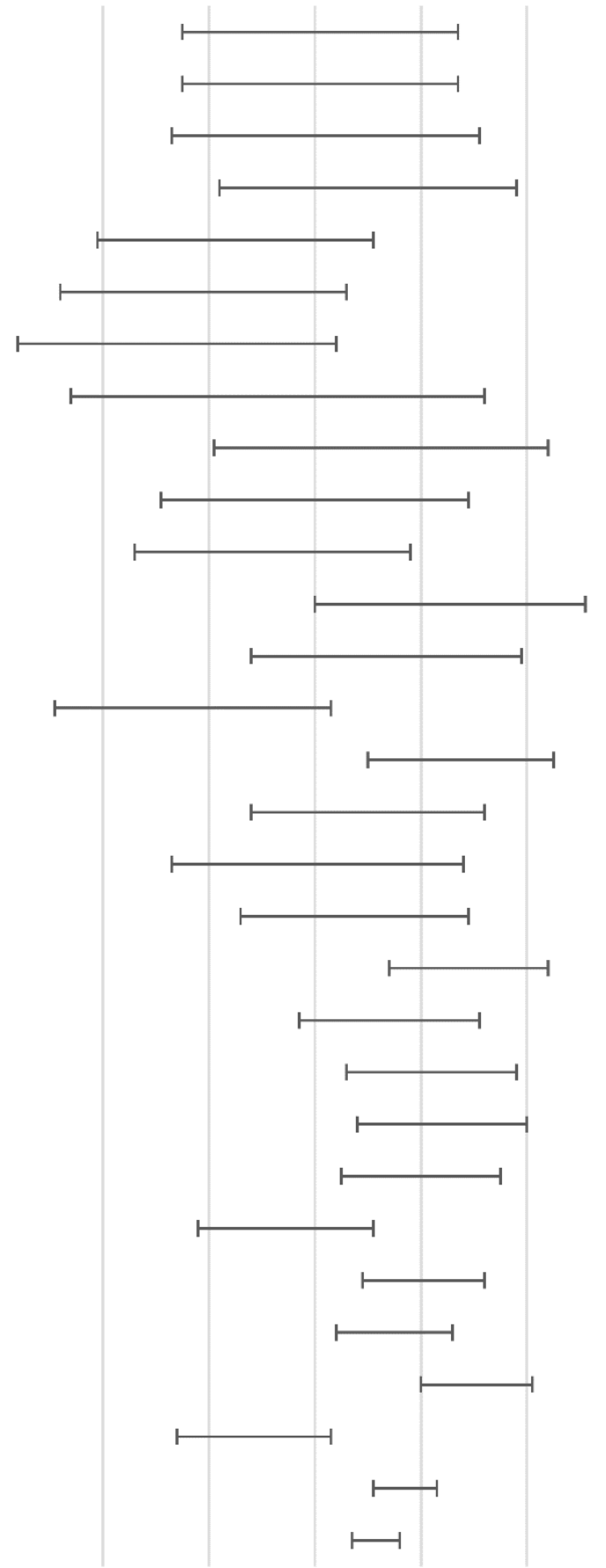

Average score

Figure 1. 95\% confidence intervals from the normal distribution formula for the average judgements on version 1 articles by the first reviewer, split by the first affiliation country (top $\mathbf{3 0}$ countries) of the first author (countries in order of increasing number of articles published).

Second, do reviewers from different countries deliver different average judgements? Reviewer judgements largely did not vary according to the location of the reviewer, with the variations found being of a level to be expected by normal statistical variations since the 
confidence intervals mainly overlap and all except two contain the 2.5 line (Figure 2). Thus, there do not seem to be international variations in the leniency of reviewers in general. There are two possible exceptions: reviewer reports from Egypt and Turkey seem to be more positive than normal. These conclusions are tentative since both are based on small sample sizes and could be influenced by non-independence in the data, such as sets of reviewers from a single department. Note also that checking confidence interval overlap is not equivalent to a hypothesis test because a small degree of overlap is consistent with rejecting the null hypothesis, and there are multiple tests, with the confidence intervals not including a correction (e.g., Sidak correction) for multiple simultaneous tests.

\section{Reviewer country}

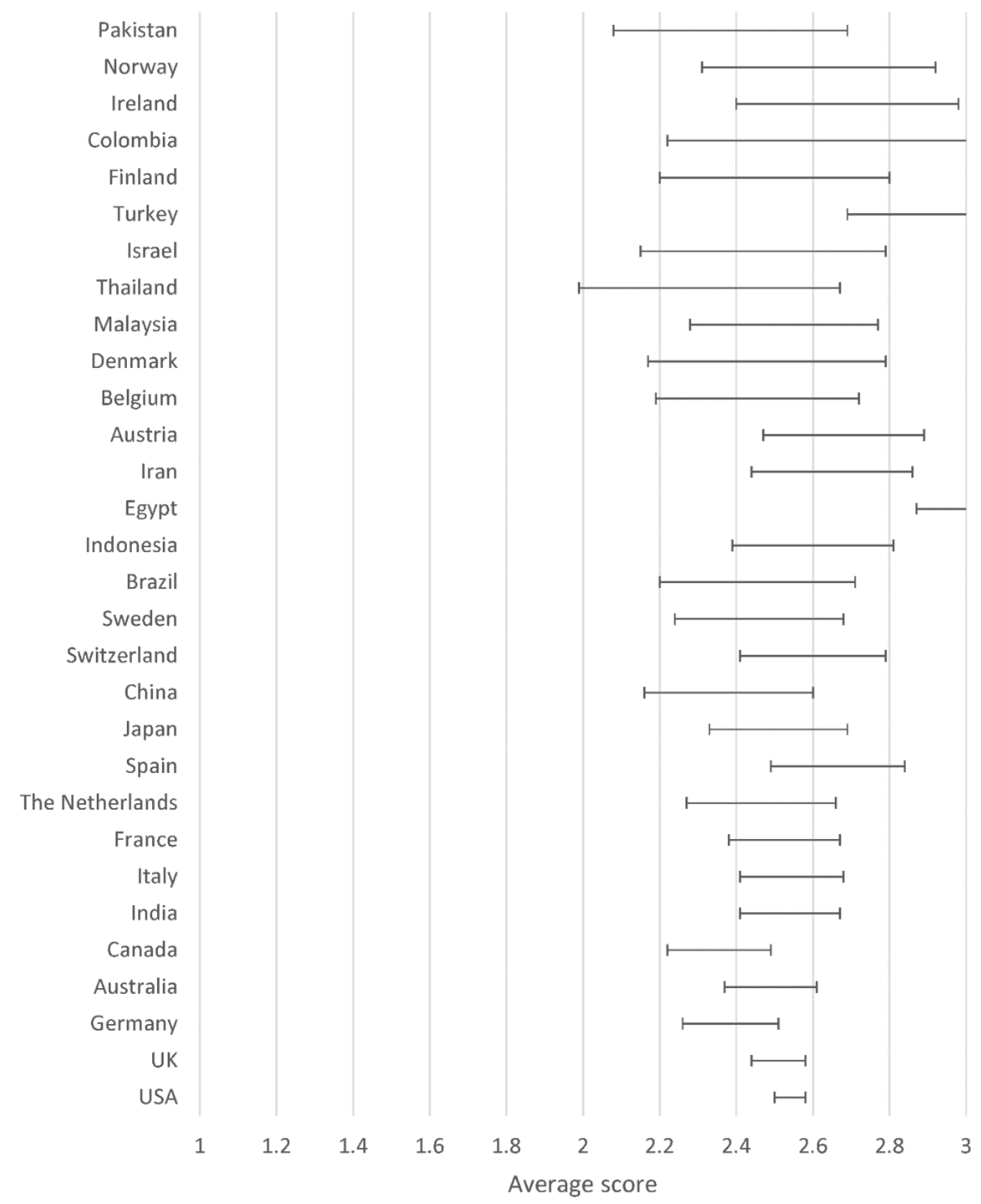

Figure 2. 95\% confidence intervals for the average judgements on version 1 articles by first reviewer affiliation country (top 30 countries) (countries ordered by increasing number of reviewers). 
Third, do countries with authors that receive more positive judgements also tend to give more positive judgements? There is a slight tendency for countries that allocate more positive judgements to also receive more positive judgements (Pearson correlation 0.13 for Figure 3; Pearson correlation 0.15 for the top 30). Although this is clearly true for Spain in contrast to Brazil, the low correlation suggests that this is not a strong international trend. The weak correlation could be due to international differences in fields submitted and reviewed for F1000Research, since there may be disciplinary differences in reviewing strictness. The positions of Spain and Brazil could be due to differing topics submitted and reviewed, for example. Similarly, it could also be a difference in the breakdown of article types received from different countries, as some article types - for example Research Articles require more stringent reviewing than others.

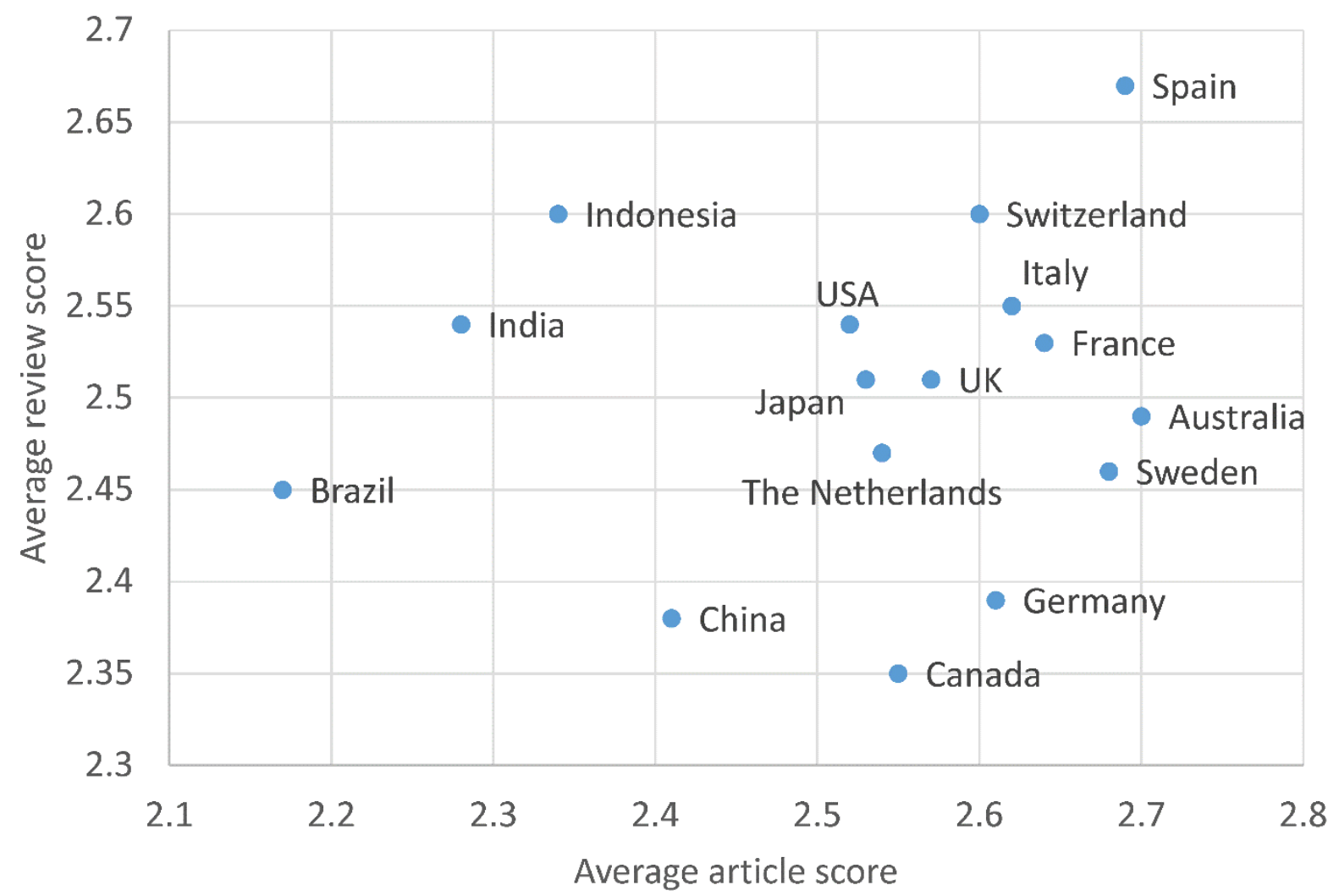

Figure 3. Average judgements on version 1 articles by first reviewer affiliation country against average article scores by first affiliation country of the first author (the 17 countries with at least 30 articles and at least $\mathbf{3 0}$ reviewers).

Fourth, do authors receive more positive judgements from reviewers with the same national affiliation? This is the main test for this section. For each country, Mann-Whitney $U$ tests were run to see whether articles tended to receive more positive judgements if the reviewer was affiliated with an institution in the same country as the first author (Table 1). A degree of same country favourable results existed for 16 of the 20 countries with the most articles, but one country, Belgium, could not be tested due to a lack of same-country reviewers. If no country had an underlying bias then the chance of 16 or more out of 19 countries giving a same-country favourable results (irrespective of statistical significance for each individual country) is 0.002 (exact Binomial test, with the null hypothesis of each country having a $50 \%$ chance of more favourable resuts from same country reviewers), giving strong 
evidence to reject the null hypothesis that there is no author-reviewer country favourable results. After a Sidak correction, the difference was statistically significant in one case, Egypt, and close to significant in one other, the UK. The slightly more powerful Benjamini-Hochberg correction method [31] would not change these results.

Reviewers were substantially more positive about Egypt-based articles if they are also based in Egypt (a score of 3: all 15 first reviewer reports from Egypt-based reviewers assigned an 'Approved' status on an Egypt-based first-authored article) compared to if the reviewer was based outside Egypt (2.29 from 23 reports). For second, third, fourth, and fifth reviewer reports, there were 14 by Egypt-based reviewers, 13 of whom assigned an 'Approved' status and one 'Approved-with-reservations'. Overall, the results suggest a community of supportive Egyptian dentistry-related researchers actively publishing in F1000Research (see Box 1). In contrast, the UK results (see Box) might be due to the country submitting strong medicinerelated articles (see Box 2).

\section{Box 1: Investigations of apparent Egyptian reviewer bias}

Only one out of 29 reports on Egypt-based author articles was assigned anything other than an 'Approved' status by an Egypt-based reviewer. Most of the author and reviewer affiliations were associated with dentistry. A possible explanation is that Egypt has excellent dentistry research, submits its high-quality dentistry research to F1000Research or has a quality control procedure in dentistry to ensure that articles are fully checked. This seems unlikely because other reviewers are less positive. For one research article, the obviously knowledgeable Egyptian reviewer recommends Approved, but the also knowledgeable UK reviewer makes a good case for Not approved (https://f1000research.com/articles/6-1786/v1). For another research article, the Egyptian reviewer report does not seem strong and the US reviewer makes a convincing case that the article should not be approved (https://f1000research.com/articles/7-1703/v1). Thus, there may be a supportive community of Egyptian dentistry researchers that express their support by not being overly critical of compatriots or by recommending less experienced colleagues as reviewers.

\section{Box 2: Investigations of possible UK reviewer bias}

Here is an example where the UK reviewer assigned an 'Approved' status and the two nonUK-based reviewers assigned 'Approve-with-reservations'. The corrections suggested by all three reviewers seem minor, so all decisions could have been Approved (https://f1000research.com/articles/6-170/v2). Another example has two UK Approveds and one non-UK Approved-with-reservations from a more detailed review, which seems reasonable (https://f1000research.com/articles/7-55/v2). Another example has one UK Approved and two non-UK Approved-with-reservations, all of which seem reasonable decisions (https://f1000research.com/articles/7-1133/v2). In all cases where a non-UK reviewer assigns 'Not approved' for an article, at least one UK reviewer also assigns 'Not approved', so there are no obvious cases of bias. Thus, there is possibly a slight tendency for UK reviewers to err on the side of generosity on the borderline of Approved and Approvedwith-reservations for articles first authored by compatriots. There were also many examples of UK articles with UK-only based reviewers, all of which assigned an 'Approved' status (e.g., https://f1000research.com/articles/7-714/v1 https://f1000research.com/articles/7$1107 / \mathrm{v} 1$ ), so it is possible that there is a medicine-related tendency for UK based authors to submit strong articles to F1000Research and suggest UK researchers as reviewers. 
Table 1: Mann-Whitney $U$ tests for same-country reviewer bias (the 20 countries with at least 20 articles). A Sidak correction gives a critical value of $p=0.0026$ to preserve the familywise error rate at 0.05 (NB no same-country reviewers for Belgium).

\begin{tabular}{|c|c|c|c|c|c|}
\hline \multirow[b]{2}{*}{$\begin{array}{l}\text { First author } \\
\text { country }\end{array}$} & \multirow[b]{2}{*}{ Articles } & \multicolumn{3}{|c|}{ Mean decision score } & \multirow[b]{2}{*}{ P* } \\
\hline & & $\begin{array}{l}\text { Same } \\
\text { country } \\
\text { reviewer }\end{array}$ & $\begin{array}{l}\text { Different } \\
\text { country } \\
\text { reviewer }\end{array}$ & Difference & \\
\hline USA & 737 & 2.53 & 2.50 & 0.03 & 0.6197 \\
\hline UK & 312 & 2.68 & 2.51 & 0.18 & 0.0077 \\
\hline India & 110 & 2.63 & 2.19 & 0.44 & 0.0147 \\
\hline Australia & 105 & 2.93 & 2.67 & 0.27 & 0.0684 \\
\hline Canada & 104 & 2.38 & 2.57 & -0.19 & 0.2976 \\
\hline Germany & 99 & 2.53 & 2.62 & -0.09 & 0.6448 \\
\hline Indonesia & 70 & 2.61 & 2.21 & 0.40 & 0.0387 \\
\hline Switzerland & 57 & 3.00 & 2.58 & 0.42 & 0.2918 \\
\hline Italy & 55 & 2.72 & 2.57 & 0.15 & 0.4917 \\
\hline France & 55 & 2.71 & 2.63 & 0.09 & 0.8269 \\
\hline The Netherlands & 52 & 3.00 & 2.50 & 0.50 & 0.0991 \\
\hline Spain & 39 & 3.00 & 2.64 & 0.36 & 0.0840 \\
\hline Iran & 38 & 2.67 & 2.30 & 0.37 & 0.1162 \\
\hline China & 32 & 2.83 & 2.31 & 0.53 & 0.1299 \\
\hline Sweden & 31 & 2.40 & 2.73 & -0.33 & 0.1638 \\
\hline Japan & 30 & 2.75 & 2.50 & 0.25 & 0.5670 \\
\hline Brazil & 30 & 2.00 & 2.18 & -0.18 & 0.6821 \\
\hline Egypt & 29 & 3.00 & 2.29 & 0.71 & 0.0024 \\
\hline Thailand & 25 & 2.40 & 2.40 & 0.00 & 0.9755 \\
\hline Belgium & 20 & None & 2.5 & NA & NA \\
\hline
\end{tabular}

*Bold values are statistically significant without a familywise error rate correction; bold underlined values are also statistically significant with a familywise error rate correction. Red values indicate a negative difference in decision scores.

\section{Does visibility and access to another reviewer's report influence subsequent reviewers?}

The second area of potential bias explored is whether through an open, non-anonymised peer review process, reviewers' decisions are influenced by the ability to view the report of another reviewer.

First, are reviewer reports more likely to refer to previous reviewer reports if the time period between the two reports being published is longer? This question needs a positive answer to justify the main test assumptions because it would indicate that a longer gap between reports associates with a greater likelihood of the subsequent reviewers having read an earlier report before publishing theirs. This was assessed with a Mann-Witney $U$ test for a difference in average time (rank) between reports for two groups:

- Group 1: The subsequent reviewer report did not mention any prior reviewer report.

- Group 2: The subsequent reviewer report mentioned a prior reviewer report.

The Mann-Whitney $U$ test found very strong evidence $(p=0.001)$ to reject the null hypothesis that there was no difference between the groups. Group 2 had higher ranks (longer time periods). Thus, there is very strong evidence that longer periods between reviewers associate 
with the subsequent reviewer being more likely to refer to a previous review. This does not prove that subsequent reviewers are more likely to have read a previous report if it was published longer ago because many reviewers may have read previous reports and not mentioned them. Nevertheless, this is a plausible hypothesis, both from common sense (as argued in the Methods section) and from this secondary test.

The median gap between reviewer reports was 13 days, with $7 \%$ being submitted on the same day and $36 \%$ during the same week. The longest gap was three years (1127 days).

Second, are reviewers more likely to mention a previous report for any outcome? A reviewer might only mention a previous report to discuss its criticisms, for example. A chisquare test was used to test for this, finding very strong evidence $(p<0.001)$ to reject the null hypothesis that there is no relationship between the decision and whether a previous report is mentioned. The main reason for this is that a reviewer report that had assigned an 'Approved-with-reservations' status was more likely to mention a previous reviewer report than was a report that had assigned an 'Approved' status (Table 2). This is likely to be because there are more potential issues for discussion and for authors to consider, or to avoid reiterating previously discussed issues.

Table 2. Chi square test for a relationship between a specific reviewer assignment and whether a previous reviewer report is mentioned. Reports on version 1 publications in F1000Research (all article types).

\begin{tabular}{|r|l|r|r|r|}
\hline \multicolumn{2}{|c|}{} & \multicolumn{2}{c|}{$\begin{array}{c}\text { Mentions a } \\
\text { previous report }\end{array}$} & \\
\cline { 3 - 5 } \multicolumn{2}{|c|}{} & \multicolumn{1}{|c|}{ No } & \multicolumn{1}{l|}{ Yes } & \multicolumn{1}{c|}{ Total } \\
\hline \multirow{2}{*}{ Not approved } & Reports & 164 & 5 & 169 \\
\cline { 2 - 6 } & Expected Reports & 161.5 & 7.5 & 169 \\
\hline \multirow{3}{*}{ Approved with reservations } & Reports & 1075 & 82 & 1157 \\
\cline { 2 - 6 } & Expected Reports & 1105.9 & 51.1 & 1157 \\
\hline \multirow{2}{*}{ Approved } & Reports & 1855 & 56 & 1911 \\
\cline { 2 - 6 } & Expected Reports & 1826.6 & 84.4 & 1911 \\
\hline Total & Reports & 3094 & 143 & 3237 \\
\hline
\end{tabular}

Third, are there variations in the time taken for a reviewer to assign a given article approval status? A Kruskal-Wallis test was used to compare the time taken to provide a peer review for the three approval statuses allowed in the F1000Research peer review model. There was a significant difference $(p=0.001)$ between decision times, with more positive approval statuses being assigned in a shorter time (Table 3). This is probably due to the challenge of securing sufficient peer reviewers to review more complex or controversial work that needs substantial reviewing effort. The time taken between an article being published and a review report appearing and it being assigned a 'Not approved' status took on average twice as long as a for an article where the report was assigned an 'Approved' status. 
Table 3. Reviewer status assignments and the time between an article and its reviews $(n=5821)$ being published (calculated separately for each review). Reports on version 1 publications in F1000Research (all article types).

\begin{tabular}{|l|r|r|}
\hline Decision & Reviews & Average (geometric mean) days to review \\
\hline Not approved & 341 & 48.6 \\
\hline Approved with reservations & 2052 & 36.8 \\
\hline Approved & 3428 & 24.2 \\
\hline
\end{tabular}

Fourth, are reviewers more likely to agree with a prior reviewer report if it is mentioned in their review? A Mann-Whitney $U$ test was used to compare the agreement rates between reviewers who did and did not mention previous reviewers. The mean ranks were almost the same, so there is insufficient evidence to reject the null hypothesis that there is no difference $(p=0.956)$. Based on the similar mean ranks, it seems unlikely that there is a substantial influence of prior reviewers on subsequent reviewers that mention their reports.

Fifth, are reviewers more likely to agree with prior reviewer reports when they have had longer to read them? This is the main test for this section. The above test is not conclusive because reviewers may avoid mentioning previous reports that they have read but disagree with. Since it is not known whether a reviewer has read a previous report, an indirect test was run, using the time between reports as an indicator of the likelihood of a reviewer having read a previous report, as argued for and tested for above (first point). A Spearman correlation test was used to compare reviewer agreement rates with the time since the previous review report. There was a statistically significant negative correlation $(-0.123, p<0.001)$, giving strong evidence to reject the null hypothesis that there is no relationship. There is thus a negative relationship: longer gaps (more time to read a review) associated with a slightly higher rate of disagreement. This is not conclusive evidence of the absence of a conformity bias from reviewers reading previous reports because the more controversial papers may require extra reviewers or may need longer for reviewing or recruitment. Nevertheless, in conjunction with the neutral result for reports mentioning previous reviewer reports, it suggests that the availability of previous reports does not produce a conformity pressure on reviewers.

\section{Conclusions}

While the use of peers as experts to provide quality assurance for scholarly output remains a cornerstone of modern science, it is known to introduce bias and is ultimately subjective. One of the arguments for the shift to more open models of peer review is that by providing comments and reviewer identities in the open, readers can consider experts' perspectives of the strengths and weaknesses of an article. Furthermore, it is argued that requiring reviewers to provide comments in the open will help to ensure that peer review is constructive, requiring reviewers to justify negative judgements, potentially reducing the scope for conscious bias.

This study found a tendency for reviewers to be more likely to assign a positive review to authors based in the same country as them (16 out of 19 countries, with one small country set statistically significant after correcting for familywise error rates). The mostly likely explanation is bias due to reviewers trying to help (or avoid animosity with) people that they are more likely to know or meet. Nevertheless, reviewers might also decline to review rather than publishing a public negative review for an acquaintance. In support of this, a study of psychology found that reviewers were more willing to sign positive reports [18], so reviewers 
may have concerns about authors knowing their identity for negative reviews. It is not known whether a location bias is common practice among authors and reviewers more generally, due to the absence of comparable data among publishers using traditional, closed peer review. Moreover, there may be similar correlations between grant applicants and their reviewers according to location (though the reverse has been found for Australian grants: [32]). Thus, whilst this study has found evidence of an affiliation bias, it is not clear whether the bias would also occur in single blind review (i.e., whether it is due to the author's affiliation being public or the reviewer's affiliation being public).

In terms of whether the ability to view the report of one reviewer exerts an influence on subsequent reviewers, there was little evidence that this is the case. It seems unlikely that the prior public availability of reviewer reports creates a pressure on reviewers towards conformity. The slight tendency towards non-conformity found in one test may well be a sideeffect of review complexity rather than a genuine non-conformity bias.

As noted above, it is important that the introduction of new approaches to peer review are tested to ensure that they do not have unintended consequences nor introduce new biases. Nevertheless, scrutiny of new processes needs to be accompanied by fuller scrutiny of established processes; we have been unable to compare our findings with the extent of bias along the dimensions we explored within other peer review models (e.g. singleblind) due to a lack of data. To be able to refine and optimise the use of experts (e.g. peer review) in grant funding and scholarly publishing requires closed peer-review data to be made available in formats that enable comparable analysis. We fully acknowledge that the findings of this study are tentative at best, weakened by our reliance upon hypotheses that cannot be fully tested and an inability to control for all relevant independent variables, such as specialist areas. A randomised controlled trial would be needed to fully assess the influence of open reviewer identities. Without this, the results may be due to unmodelled variables, such as international differences in the specialties of articles (for national affiliation bias) and the possibility that reviewers tend to ignore published reports that they disagree with (for prior report publication bias).

We hope that this analysis will contribute to the evidence base and decision-making about how and where open peer review can be used to best effect. We particularly hope to see a growth in studies that aim to understand how the processes and workflows used to support research can influence the outcomes of research itself.

\section{References}

1. Lee CJ, Moher D. Promote scientific integrity via journal peer review data. Science. 2017; 357(6348): 256-257.

2. Morey RD, Chambers CD, Etchells PJ, Harris CR, Hoekstra R, Lakens D, Vanpaemel W. The peer reviewers' openness initiative: Incentivizing open research practices through peer review. Royal Society Open Science. 2016; 3(1): 150547. https://doi.org/10.1098/rsos.150547

3. Ross-Hellauer T. What is open peer review? A systematic review [version 2; peer review: 4 approved]. F1000Research. $4017 ; \quad 6: 58$. doi:10.12688/f1000research.11369.2

4. Siler K, Lee K, Bero L. Measuring the effectiveness of scientific gatekeeping. Proceedings of the National Academy of Sciences. 2015; 112(2): 360-365. 
5. Ceci SJ, Williams WM. Understanding current causes of women's underrepresentation in science. Proceedings of the National Academy of Sciences of the United States of America. 2011; 108(8): 3157- 3162.

6. Fox CW, Burns C S, Meyer JA. Editor and reviewer gender influence the peer review process but not peer review outcomes at an ecology journal. Functional Ecology. 2016; 30(1): 140-153.

7. Tamblyn R, Girard N, Qian CJ, Hanley J. Assessment of potential bias in research grant peer review in Canada. CMAJ. 2018; 190(16): E489-E499. doi: 10.1503/cmaj.170901. PMID: 29685909; PMCID: PMC5915246.

8. Witteman $H$, Hendricks $M$, Straus $S$, Tannenbaum $C$. Are gender gaps due to evaluations of the applicant or the science? A natural experiment at a national funding agency. The Lancet. 2019; 393(10171): 531-540

9. Wang $Q$, Sandström $U$. Defining the role of cognitive distance in the peer review process with an explorative study of a grant scheme in infection biology. Research Evaluation. 2015; 24(3): 271-281.

10. Harris M, Macinko J, Jimenez G, Mahfoud M, Anderson C. Does a research article's country of origin affect perception of its quality and relevance? A national trial of US public health researchers. BMJ Open. 2015; 5(12): e008993.

11. Peters DP, \& Ceci SJ. Peer-review practices of psychological journals: The fate of published articles submitted again. Behavioral and Brain Sciences. 1982; 5(2) 187-195.

12. Rodríguez-Bravo B, Nicholas D, Herman E, Boukacem-Zeghmouri C, Watkinson A, Xu J, Abrizah $A$, Świgoń $M$. Peer review: The experience and views of early career researchers. Learned Publishing. 2017; 30(4): 269-277.

13. Moylan EC, Harold S, O'Neill C, Kowalczuk MK. Open single-blind double-blind: which peer review process do you prefer? BMC Pharmacology and Toxicology. 2014; 15: 55. doi:10.1186/2050-6511-15-55

14. Mulligan A, Hall L, Raphael E. Peer review in a changing world: An international study measuring the attitudes of researchers. Journal of the American Society for Information Science and Technology. 2013; 64(1): 132-161.

15. Ross-Hellauer T, Deppe A, \& Schmidt B. Survey on open peer review: Attitudes and experience amongst editors authors and reviewers. PloS One. 2017; 12(12) e0189311.

16. Van Rooyen S, Godlee F, Evans S, Black N, Smith R. Effect of open peer review on quality of reviews and on reviewers' recommendations: a randomised trial. Bmj. 1999; 318(7175): 23-27.

17. Van Rooyen S, Delamothe T, Evans SJ. Effect on peer review of telling reviewers that their signed reviews might be posted on the web: randomised controlled trial. Bmj. 2010; 341: c5729.

18. Walsh E, Rooney M, Appleby L, Wilkinson G. Open peer review: a randomised controlled trial. The British Journal of Psychiatry. 2000; 176(1): 47-51.

19. Jefferson $T$, Alderson $P$, Wager $E$, Davidoff $F$. Effects of editorial peer review: a systematic review. Jama. 2002; 287(21): 2784-2786.

20. Bravo G, Grimaldo F, López-Iñesta E, Mehmani B, Squazzoni F. The effect of publishing peer review reports on referee behavior in five scholarly journals. Nature Communications. 2019; 10: 322

21. Walker R, Barros B, Conejo R, Neumann K, Telefont M. Personal attributes of authors and reviewers social bias and the outcomes of peer review: a case study [version 2; 
peer review: 2 approved]. F1000Research. 2015; Available at: https://doi.org/10.12688/f1000research.6012.2

22. Kirkham J, Moher D. Who and why do researchers opt to publish in post-publication peer review platforms? findings from a review and survey of F1000 Research [version 1; peer review: 2 approved 1 approved with reservations]. F1000Research. 2018; Available at: https://f1000research.com/articles/7-920/v1

23. Brand A, Allen L, Altman M, Hlava M, Scott J. Beyond authorship: attribution contribution collaboration and credit. Learned Publishing. 2015; 28(1): 151-155. doi:10.1087/20150211

24. Larivière $V$, Desrochers $N$, Macaluso $B$, Mongeon $P$, Paul-Hus $A$, Sugimoto $C R$. Contributorship and division of labor in knowledge production. Social Studies of Science. 2016; 46(3): 417-435.

25. Barros T Allen L. Assessment of agreement between two reviewers in the open postpublication peer review process of F1000Research [version 1; not peer reviewed]. F1000Research. 2017;

6:1678(poster), https://doi.org/10.7490/f1000research.1114870.1

26. Björk BC. Acceptance rates of scholarly peer-reviewed journals: A literature survey. El Profesional de la Información. 2019; 28(4): e280407.

27. Sugimoto CR, Larivière $\mathrm{V}$, Ni C, Cronin B. Journal acceptance rates: a cross-disciplinary analysis of variability and relationships with journal measures. Journal of Informetrics. 2013; 7(4): 897-906.

28. Smith MJ, Weinberger C, Bruna EM, Allesina S. The scientific impact of nations: Journal placement and citation performance. PloS One. 2014; 9(10): e109195.

29. Thelwall M, Levitt JM. National scientific performance evolution patterns: retrenchment successful expansion or overextension. Journal of the Association for Information Science and Technology. 2018; 69(5) 720-727.

30. Xie Y. Sociology of science. "Undemocracy": inequalities in science. Science. 2014; 344(6186): 809-810. doi:10.1126/science.1252743

31. Benjamini Y, Hochberg Y. Controlling the false discovery rate: a practical and powerful approach to multiple testing. Journal of the Royal statistical society: Series B (Methodological). 1995; 57(1): 289-300.

32. Marsh HW, Jayasinghe UW, \& Bond NW. Improving the peer-review process for grant applications: reliability validity bias and generalizability. American Psychologist. 2008; 63(3): 160. 